\title{
Revisiting pest sampling plans in light of economic uncertainty and risk aversion
}

Diego F. Rincon ${ }^{1 *}$, Hugo Fernando Rivera-Trujillo ${ }^{1}$, Lorena Mojica-Ramos ${ }^{1}$, Felipe BorreroEcheverry $^{1}$

${ }^{1}$ Corporación Colombiana de Investigación Agropecuaria - AGROSAVIA. Centro de Investigación Tibaitatá, Km 14 vía Mosquera-Bogotá, Mosquera, Colombia

* Corresponding Author

Email addresses: drincon@agrosavia.co (DFR), fborrero@agrosavia.co (FB-E), hrivera@agrsavia.co (HFR-T), smojica@,agrosavia.co (LM-R) 


\section{Abstract}

2 Decision-making for pest management in agriculture is often assisted by sampling plans that guide

3 users in determining the need for an intervention. Even though Tuta absoluta is easily recognizable

4 by most tomato growers and that several sampling plans have been developed, adoption of decision-

5 making systems for this pest is still incipient. Two potential obstacles for adoption are market

6 uncertainty and farmer's risk aversion. Both obstacles could be tackled by adopting sampling plans

7 that allow farmers to plan interventions according to rough estimations of economic thresholds and

8 the intuition and experience gained by farmers. In this study, we evaluated four sampling plans

9 using computer simulations and field trials. We compared the efficiency and the ability of each plan

10 to both estimate the actual mean number of larvae per plant and to classify pest populations

11 according to a predefined economic threshold. We also analyzed the time spent, and plants

12 examined by human subjects applying each plan on a tomato crop with a T. absoluta infestation

13 slightly over a predefined economic threshold. We show that sampling plans that deliver the most

14 precise classifications, are poorest in delivering pest density estimations and vice versa. Our

15 findings are consistent for both human subjects and computer simulations. However, the average

16 number of samples required by sampling plans does not reflect the time spent by humans sampling

17 real plants. Our results show that sampling plans based on counts, as opposed to those based on

18 binary data, can efficiently provide reliable information on a current level of T. absoluta infestation

19 relative to an estimated decision threshold. We suggest that sampling plans that promote the

20 creation of farmer's memory, such as those based on counts, may be more suitable to both reduce

21 risk aversion and increase adaptability to market uncertainty.

22 Keywords: Tomato leafminer, Tuta absoluta, pest monitoring, integrated pest

23 management, economic injury level, decision-making, dynamic threshold, information-

24 based agriculture 


\section{1. Introduction}

26 The on-going digital agricultural revolution aims to tackle unsustainable agriculture through the use

27 of biopesticides and biofertilizers, ecosystem services, and the use of information (Fraser and

28 Campbell 2019). While the use of bioproducts helps to replace chemical inputs, and ecosystem

29 services helps to reduce the need of corrective treatments, information plays a key role in decisionmaking (Barzman et al. 2015). Market prices, changing customer demands, and policies are ever looming risks which farmers face. These risks reduce the willingness of farmer to assume others, such as insect pests, which they can control using unsustainable (though immediately effective) practices. As such, effective procedures that help farmers make informed decisions on pest management are fundamentally linked to the adoption of Integrated Pest Management (IPM) programs, and favor intrinsic system controls over corrective interventions, which reduces costs and detriments to the environment and public health (Peterson et al. 2018).

Conventionally, sequential sampling plans have been the most widely used in pest management and are often developed for either estimation or classification. Sequential sampling for estimation has been used mainly for research purposes because they aim to deliver population density estimations at predefined (fixed) precision levels, at the cost of being labor intensive. On the other hand, sequential sampling for classification has been used more widely in decision making since it

42 requires fewer samples and delivers a categorical recommendation (intervene or not) based in pre-

43 defined thresholds. Both estimation and classification sampling may be based on binomial

44 assessments or on discrete counts. The former is clearly faster and easier to carry out but lacks the 45 precision of the later. Sequential binomial sampling plans, based on the sequential probability ratio tests (SPRT) framework (Wald, 1945), became popular for decision-making in pest management because they require relatively low number of samples, and data collection is usually feasible (at least for low tally thresholds). However, pest population classification by sampling based on fixed economic thresholds is often unrealistic, given the significant uncertainty about produce prices and control costs (Rincon et al. 2019; Plant 1986). There is also the potential of continuously making biased decisions if the sampling unit is chosen too obvious to classify, because users might systematically select positives (or negatives). Moreover, requiring a low number of samples might not necessarily lead to a substantially lower sampling effort and farmers may be open to examining

54 a reasonable number of samples as long as they can ensure sound management decisions.

55 The tomato leaf miner (TLM), Tuta absoluta (Meyrick) (Lepidoptera: Gelechiidae), is one of the 56 most limiting pests of greenhouse and open field tomatoes in most producing regions of the world.

57 Larvae usually mine leaves but may occasionally cause direct damage to fruits (Figure 1). The 
reason why some larvae prefer to feed on fruits over leaves is unknown, but there are some reports claiming that such preference is density-dependent (Cassino et al. 1995; Cocco et al. 2015). Since larvae damaging fruits cause most economic losses, economic thresholds for this pest are based on estimations of larval or mine density as a proxy for proportion of infested fruits (e.g., Cocco et al. 2015). Although the economic effect of moderate densities of the TLM is probably negligible (up to $73 \%$ of fully-formed leaves with $>0$ larvae are required to cause economic damage (Cocco et al. 2015), farmers tend to have a zero-tolerance for this pest. In south America, reports indicate that farmers use up to 36 applications of broad-spectrum chemical insecticides in a single crop cycle to prevent damage from the TLM, most of which are probably unnecessary (Donado et al. 2017). This overuse of insecticides has resulted in the development of resistance to most registered molecules (Haddi et al. 2012; Silva et al. 2011).

Even though $T$. absoluta is easily recognizable by most tomato growers and that some sampling plans have been developed, adoption of decision-making systems for this pest is still incipient, particularly in developing countries(Parsa et al. 2014). Two potential reasons are (1) the high startup investment (greenhouse facilities and elevated production costs) that makes tomato farmers particularly risk-averse, and (2) the difficulty to estimate reliable economic thresholds, given the uncertainty in tomato market price (which is often outside of their control) . Both, market uncertainty and risk aversion could be dealt with by providing farmers with means to experiment, learn and adapt from small, informed (calculated) experiments (Feola and Binder 2010). In fact, human decision-making is known to rely mostly on intuition and on feelings of "what seems to be correct", rather than on complicated calculations or normative rules, especially under risky situations (Tversky and Kahneman 1981; Barberis 2013). For example, sampling plans that provide reliable indications of how close a pest population is from reaching a rough estimate of the economic injury level (EIL) could allow farmers make their own predictions and corresponding calculated bets. In the long-run, it is expected that both, reliable information provided by sampling plans and farmers' intuition and experience on reducing economic risks associated with produce prices will increase adaptation to uncertainty.

In this study, we evaluated two sequential and two variable-intensity sampling (VIS) plans for classification using computer simulations and field trials. We decided to exclude sampling plans for estimation from our study, since our focus is sampling based on pre-defined thresholds for decisionmaking. We compared the efficiency and the ability of each plan to both estimate the actual mean number of larvae per plant and to classify pest populations according to a predefined economic threshold. We also analyzed the time spent, and number of plants examined by human subjects 
91 while applying each plan on a tomato crop with a T. absoluta infestation slightly over a predefined

92 economic threshold. We show that classification might not always be the correct goal, especially

93 when the pest population is near the threshold, and that the mean number of samples required to

94 make classifications is not always related with the time spent by human subjects collecting and

95 recording the samples. We propose that sampling plans should be selected based on the level of

96 economic uncertainty and that management decisions should be made by farmers based on results

97 of sampling and their willingness to assume calculated risks. Only through the use of reliable

98 information will farmer's perception of risks change, allowing them to tolerate pests at acceptable

99 levels in their crops, reduce pesticide use and move towards a more sustainable future.

100

101

102

103

104

105

106

107

108

109

110

111

112

113

114

115

116

117

118

119

120

121

\section{Materials and methods}

Eight tomato greenhouses of approx. $3000 \mathrm{~m}^{2}$ from two contiguous municipalities, Santa Sofía and Sáchica (Boyacá, Colombia) were sampled five times between November 2017 and July 2018 to obtain 40 datasets. Each dataset consists of approx. 100 samples, which were collected from sampling units that were distributed uniformly (in an equidistant grid) within each greenhouse. In order ensure moderate natural TLM infestations to occur in the selected greenhouses, formal agreements with farmers were made in which an economic compensation was offered in exchange for delaying or suppressing insecticide applications that may hamper pest occurrence or spatial distribution. To homogenize sampling size across events, sampling units were defined based on crop age as follows: 1-50 days after planting (dap), 3 plants; 51-90 dap, 2 plants, 90-130 dap, 1 plant. Variances were modeled as a function of means using least squares and the linearized version of Taylor's Power Law (TPL), which is defined as:

$\log \left(\sigma^{2}\right)=\log (a)+b \log (\mu) \quad($ Eq. 1)

where $\sigma^{2}$ is the sample variance associated with the mean, $\mu$, number of larvae per sample unit, and $a$ and $b$ are parameters.

We selected two modalities of sequential plans, the SPRT (Wald 1945) and VIS (Hoy et al. 1983; Hoy 1991); and two different strategies for data collection, by counts, and by presence/absence in sampling units (binomial). Briefly, SPRT plans rely on sequential likelihood ratio tests made each time a sample is collected until there is enough evidence to decide a classification with a predefined probability of misclassification. The VIS plans, in turn, require an extensive examination of the entire field, so a predefined number of transects are distributed systematically throughout the field. The idea is to examine all the transects with a varying level of intensity (number of subsamples) 
122 according to a required precision, which is calculated as a function of sequential estimations of the

123 pest population density. The sampling plans were developed using the equations and procedures

124 described in Binns et al. (2000), including the empirical model used to predict the proportion of

125 infested plants (with $>1$ larva), $P_{T}$, as a function of the mean number of larvae per plant, $\mu$ :

$126 P_{T}=1-\exp \left(-e^{c} \mu^{d}\right)$

(Eq. 2),

127 where $c$ and $d$ are parameters, which where estimated from data by least squares using the linear 128 version of Eq. 2:

$\ln \left[-\ln \left(1-P_{T}\right)\right]=c+d \ln (\mu)$

(Eq. 2a).

130 Evaluation of sampling plans was carried out using two different strategies. The first one consisted 131 of the conventional approach by simulating sampling events with computer generated data. The 132 negative binomial distribution was used to model the distribution of larval counts, and as the 133 reference for data generation. The aggregation parameter, $k$, used for simulations was calculated as 134 a function of the mean number of larvae per plant:

$135 \quad k(\mu)=\frac{\mu^{2}}{a \mu^{b} e^{z}-\mu}$ (Eq. 3),

136 where $z$ is a random normal variate with zero mean and standard deviation, $\sigma_{\varepsilon}$, which equals the 137 mean squared error of the regression model used to fit Eq. 1. Each time the counts of a TLM 138 population are generated with a predefined mean $(\mu), k$ is assigned with a different value according 139 to the uncertainty about $a$ and $b$ (Binns et al. 2000).

140 Data was generated using an algorithm that included a random number generator in a negative 141 binomial and Eq. 3 to assign values for the aggregation parameter, $k$. Validation was performed by 142 comparing generated with observed counts, which came from the 40 datasets that were collected 143 from the eight greenhouses in the study area. One thousand simulations were generated for each 144 observed mean, and the mean probabilities for each value were compared with observed probability 145 distributions by chi-squared tests. The precision level was calculated as the proportion of observed 146 probability distributions not significantly different from the simulated distributions over the total 147 datasets.

148 Using simulations (in silico), both the operating characteristic (OC) average sample number (ASN) 149 functions were constructed for each sampling plan. In addition, accuracy functions were derived by 150 estimating the root mean squared error (RMSE) associated to a range of larval densities. This 151 association allows the evaluation of sampling plans for their ability to estimate pest densities as they 152 approach a predefined threshold. This measure is useful in deciding which sampling plan is best in 
situations when there is uncertainty about the predefined threshold. We used a standardized version of the RMSE:

$$
R M S E=\sqrt[2]{\frac{\left(\mu_{o}-\mu_{e}\right)^{2}}{N}} / \mu_{e}
$$

where $\mu_{o}$ represents the means (larvae/plant) obtained through the sampling plans, $\mu_{e}$ the real means, and $N$ the number of simulations. Lower RMSE values denote higher accuracy. All three, OC, ASN and RMSE functions where constructed with 1000 simulations for each pest density in a range of 0-20 larvae per plant.

In addition to the simulations, a field trial (in situ) was carried out to evaluate the sampling plans in a real tomato crop with real human subjects. A greenhouse tomato crop (Solanum lycopersicum L. var. Libertador) of approx. $320 \mathrm{~m}^{2}$ and 760 plants was planted using conventional procedures similar to those used by growers in the study area. Plants were distributed in eight rows of 95 plants each, with $1.2 \mathrm{~m}$ between rows and $30 \mathrm{~cm}$ between plants within the same row. Two artificial infestations of 450 TLM adults took place, one at 73 dap and the other at 80 dap (900 adults total). In order to establish a golden standard to compare with the results delivered by the evaluated sampling plans, an intensive sampling event was carried out in which 101 plants ( $\sim 12$ per row) homogenously distributed in space were examined and the number of larvae in each was registered. Once the golden standard was estimated, the predefined threshold was set slightly below it, in such a way that the threshold upper limit equals the golden standard 95\% confidence interval lower limit. This threshold was used for both, the in silico and in situ experiments and does not necessarily reflect a real economic threshold, since it was set solely for evaluation purposes.

The field trial was carried out by comparing the performance of the four sampling plans when they were conducted by eight human subjects. Each human subject reported the final decision (intervene or not), and the number of samples and time required to make that decision. The mean number of larvae reported by the human subjects was compared with the golden standard, as well as the proportion of correct decisions and the mean time spent by users conducting each sampling plan. All analyses were carried out using software R (version 3.5.1) (R Core Team 2018).

\section{Results and discussion}

The means observed in the 40 datasets collected in the field ranged between 0.009 and 33.287 larvae per sampling unit, while the observed variance varied between 0.009 and 192.337. The TPL described well the variance-mean relationship of larval counts $\left(P<0.001, \mathrm{n}=40, F=1249, \mathrm{R}^{2}=\right.$ 
0.970) (Figure 2a). Parameter estimate for $a$ was $1.830 \pm 0.051$ (estimate \pm standard error), and $1.218 \pm 0.034$ for $b$. We also found good fit between Eq. 2 and the proportion of infested plants explained as a function of the mean number of larvae per plant $\left(P<0.001, \mathrm{n}=40, F=1102.9, \mathrm{R}^{2}=\right.$ 0.967) (Figure 2b). The parameter estimate for $c$ was $-0.384 \pm 0.036$, and $0.844 \pm 0.025$ for $d$. Altogether, the algorithm developed to simulate TLM larvae counts showed that $92 \%$ of the observed probability distributions was not significantly different from the simulated distributions $(P$ $>0.1$ ). Such precision level could be considered adequate for the purpose of this research (Rykiel 1996).

Through the intensive sampling in the experimental crop, the estimated $95 \%$ confidence interval of mean number of larvae per plant was $[10.647,14.878]$ (mean $=12.762)$, which was used as golden standard for comparing sampling plans. Thus, we set the decision threshold interval at $[8,10]$ larvae per plant (mean $=9$ ), which is equivalent to $\sim 98-99 \%$ of plants infested (with $>0$ larvae), according to Eq. 2. We think that such a threshold, although apparently high (relative to that established by farmers), might be close to the real one, based on ongoing field studies (Rincon et al., unpublished results). Moreover, Cocco et al. (2015) estimated that between 47 and 73\% of fully formed leaves infested are required to cause $3 \%$ of damaged fruits, which might be close to our designated threshold (98-99\% of plants infested), considering the aggregated nature of TLM larvae within plants (Torres et al. 2001).

Simulations revealed that sampling plans differed considerably in their ability to deliver accurate recommendations (Figure 3a-b). According to the OC functions, the sampling plans based on counts produce less incorrect decisions than those based on presence/absence (binomial). In general, all four plans are conservative, since most mistakes are made in the area of applying an intervention when it was not really needed (Figure 3a). However, binomial sampling plans tend to deliver incorrect recommendations almost exclusively when pest population is under the decision threshold, while plans based on counts showed a more balanced trend. Such unbalanced misclassification trend showed by binomial plans could be a consequence of the relationship between mean number of larvae and proportion of plants infested, which is relatively insensitive when means fall above $\sim 6$ (Figure $2 b$ ). So, when populations are high, the proportion of plants infested does not seem to change enough to allow binomial samplings distinguish populations that are below the threshold.

According to the simulations, in general, SPRT plans required less samples than VIS plans. This is not surprising since VIS plans, in contrast to SPRT plans, require the user to examine a predefined number of transects irrespective to how far or how close the estimated pest density is from the threshold. By far, the VIS binomial plan required the greater number of samples, especially when 
pest population is above the designated threshold (Figure 3c-d). This effect might be a consequence of repeated sequential estimations of proportions that are very close to the threshold, which cause the maximum sampling intensity to be required in most transects. Again, binomial plans (SPRT and VIS) showed a distinct pattern, since the required sample size does not decrease even when pest population is way above the threshold. Presumably, the proportion of infested plants is so similar at high population densities (approaching 1) that binomial classifications tend to demand a maximum number of samples to distinguish between populations that are above or below the threshold.

Among the four sampling plans, according to the simulations, the VIS plans produced smaller RMSE than SPRT plans, and VIS by counts evidenced the greatest accuracy on estimation (Figure 3e-f). In general, sampling plans based on counts were more accurate estimating TLM population density than those based on binary data. This is not surprising since accuracy reflected the sample size required by each sampling plan. It is interesting that those plan that excel in pest classification accuracy are those that perform poorly in estimation and vice versa.

We found similar results in the field trials with human subjects. Sampling plans based on counts were more accurate estimating TLM population density than those based on binary data. Also, similar to what we observed in simulations, the SPRT binomial was the most inaccurate plan for estimation purposes (highest RMSE). However, among plans based on counts, the VIS had slightly higher RMSE than the SPRT (Figure 4a-b), which contrasts with what was found with simulations (Figure 3f). This apparent contradiction could be due to the high variability in estimation of means for VIS plans, relative to the variability observed for the SPRT plan (Figure 4a). This difference in variability was not observed in simulations, probably because number generation in simulations was not spatially explicit and VIS sampling, in contrast to SPRT plans, is highly sensitive to the place chosen to collect the first sample. Thus, the number of samples required by VIS plans varied in the field trial as a result of differences in estimation after the first few samples were collected (participants were allowed to select the location of the first sample freely). Such variation is reduced in simulations since each time a sampling was simulated, a new dataset was generated, without considering spatial variations explicitly.

The mean number of samples required to make a decision that human subjects reported followed a similar trend in comparison with what was observed in simulations (Figure 4c-d), where VIS and counts demanded more samples than SPRT and binomials, respectively. In fact, except for the VIS binomial, the required mean number of samples for each sampling plan was similar in the field trial and in the simulations performed for pest densities of 12 larvae/plant $(\sim$ pest density found in the intensive sampling in the field), which were $6.87 \pm 0.81$ larvae/plant and $10.96 \pm 0.22$ for SPRT 
counts, $30.3 \pm 8.98$ and $27.55 \pm 0.50$ for VIS counts, $47.87 \pm 5.95$ and $300.57 \pm 2.45$ for VIS binomial, and $27.66 \pm 2.81$ and $30 \pm 0$ for SPRT binomial, for observed and simulated data, respectively. It is interesting that all human subjects reported a "no intervene" decision when they performed the VIS binomial plan, even though the resulting estimation of the population density was even better than that delivered by the SPRT binomial, which correctly released the recommendation of "intervene" $100 \%$ of the times. It is likely that the unusually high mean number of samples found in simulations for the VIS binomial plan is the result of an artifact produced by the lack of spatial structure in the procedure for number generation in the simulation algorithm.

Despite the robust trend found in the mean number of samples required by each sampling plan, such differences among plans were not reflected in the time human subjects spent performing them. According to the time elapsed recorded by the participants, all plans seemed to require roughly the same amount of time (Figure 4d). One exception could be the SPRT by counts, which might require less time in average than VIS by counts.

We show that the evaluated sampling plans differ in their ability to both estimate pest population size and deliver accurate management recommendations. According to our results, binomial sampling plans perform best when the purpose is to deliver a recommendation based in a fixed control threshold. However, binomial sampling plans loose accuracy in estimation when populations are high, whereas sampling methods using counts remain robust independent of the population density (Figure 3e). In fact, we argue that, in practice, binomial methods, particularly SPRT, may be overestimating populations when populations are low since real randomization of observations is rare and will tend to be skewed when damage is highly conspicuous (as was our case), an effect that we cannot observe in simulations. Methods based on counts, on the other hand, may be limited by the amount of time (labor) needed in cases when pests may be too numerous or difficult to count (such as whitefly or mites). While this may be true, we do not see a significant decrease in the time spent between plans using counts and those based on binary data, although this is likely to change among systems and crops of different areas. Similarly, VIS plans performed best estimating TLM population size than did SPRT plans, but the accuracy of the final recommendation did not change with the type of the plan. SPRT plans required a smaller number of samples to reach a decision than VIS plans, which was not surprising given that SPRT were originally developed for quality control applications (Wald 1945), while VIS was designed for pest management (Hoy et al. 1983). VIS plans have the advantage of requiring that growers walk through the entire crop which allows them to simultaneously observe the plants for other problems and obtain empirical information that can be used to focus pesticide applications only where it is required. A potential 
limitation of VIS plans is that they require a constant recalculation of subsequent sample size, which is hardly realistic since farmer could not be expected to run these calculations as part of their daily routine. However, with the computing power that we all carry in pockets nowadays, applications to that carry out these calculations may facilitate the uptake and implementation of VIS as a strategy. As a matter of fact, the human subjects who evaluated the sampling plans in the field trial used a simple R script on their phones to implement both VIS plans (counts and binomial).

To a great extent, the whole idea behind developing and implementing sampling plans based on EILs is to favor the adoption of IPM programs and hence the reduction of the dependence on chemical inputs. However, technologies that objectively guide the need of insecticide applications are not widely adopted by farmers, especially in the developing world (Parsa et al. 2014; Morse 2008). We identify two general obstacles for this lack of adoption. The first is the uncertainty about the establishment of decision thresholds, which comes mainly from produce market volatility, and variations in cost and level of efficacy of control measures (Naranjo et al. 1996; Plant 1986). The relative impact of each depends on the system, but the globalization of markets has particularly increased the risk associated with the income farmers can obtain from their crops, which is often more volatile than the reported produce price in the market. Although the usefulness of control thresholds has never been to achieve an economic optimum, even rough estimates of fixed (nominal) economic thresholds assuming stable markets seem to be unrealistic. However, many farmers have acquired the skills to make good guesses on the prices they can expect in a given time, based on weather, experience, or behavior of competitors (Stonehouse 1995). In fact, Stonehouse (1995) suggests that, in the developing world context (i.e., small farms and ecogeographical heterogeneity), experience associated with local production systems might be more important than advises from extension services when establishing decision criteria for pest problems. As we previously suggested (Rincon et al. 2019), the promotion of marketing information systems that allow farmers to adapt to market uncertainty might be one way to reduce vulnerability due to market uncertainty and increase their tolerance to pest populations accordingly.

A second obstacle we devise is the inherent risk aversion of farmers, and the myriad ways they can alter their behavior in response to additional information. In general, one should expect that the more information about the risks, the higher the rationality associated with the decision-making process, as judgement is refined based on better calculation of true outcome probabilities (Pratt et al. 1995). This should be especially true under conditions of heightened uncertainty, which is when people often have more difficulty making decisions. However, this is not always the case in human behavior. When the risk estimated from outsiders (e.g., decision-support systems, neighbors, 
extensionist, pesticide salesmen, etc.) is divergent, farmers may respond irrationally by asymmetrically weighing information sources, increasing the influence of sources, which predict higher risk. For example, Lybbert et al. (2016) found an overall negative environmental impact of a disease forecast system to assist wine grape farmers' management decisions. Apparently, rather than adjusting their reaction proportionally to the estimated risk, farmers responded by applying asymmetrically more aggressive control treatments as the estimated risk from the forecast system increased. When people are exposed to multiple and conflicting risk estimations, they tend to

322 disproportionately weight sources that indicate the highest risk. This effect is referred to as the

323 "alarmist decision" and results in both an excessive consideration of worse case scenarios in

324 decision-making processes and increased risk aversion in learning. Unfortunately, this distortion in

325 judgment is observed regardless the level of credibility of the information source, and it is the mere

326 divergence in risk estimations from different sources that triggers it (Viscusi 1997). In other words,

327 decision-making in pest management seems to be stuck in risk aversive behavior as long as farmers are subjected to divergent risk estimations (which is the most common scenario). However, according to recent theoretical developments a one way to overcome this apparent trap is to focus on farmer experimentation as the foundation of the decision-making process. Keller et al. (2019) show that even the most risk-averse individuals might make risky decisions when risky situations produce information that can be used to reduce risk in the future. We advocate that sampling plans for decision-making in pest management should offer a focus on experimentation, in such a way that farmers are provided with tools to make calculated bets (experiments) on the establishment of action thresholds. For example, sampling plans, such as VIS by counts -as shown in this study-, can provide reliable information on the status of a tomato crop in relationship with a rough estimate of a predefined threshold. Considering the uncertainty associated with the management decision, the farmer may use the estimation of how close the pest population is from the estimated threshold to take calculated risks and collect valuable information, initiating an experience-based learning process, that can be used to make better decisions during future crop cycles at the cost of an

341 investment in sampling time.

342 Our results show that sampling plan modalities that are already developed could be used to reduce 343 vulnerability of production systems and potentially increase adoption rate of decision-making 344 systems by including the opportunity to learn as part of the output. This is especially important for 345 systems like the TLM in greenhouse tomato crops, where current risk perception is considerably 346 high relative to the real effect of moderate infestations on yield. Such aversion can be dealt with by providing the appropriate tools to allow farmers to make rough but sound estimates of economic thresholds, taking into account predictions of produce prices and costs of control measures. Once 
the threshold is estimated, the selection of the sampling plan should be based on obtaining information about the infestation status relative to the estimated threshold, and not solely on reducing the effort required. We propose that VIS plans, especially by counts, are the best suitable for this purpose, at least for TLM management in greenhouse tomato crops. VIS plans delivered the most precise estimations, while requiring reasonable time and effort. Such estimations are to be used as a guide to allow farmers decide which action is most appropriate for the given context (tomato price volatility, time to harvest, pest population size, etc.) rather than as management recommendation. Of course, implementation of adaptive sampling plans, such as those suggested in this research, require simultaneous technological developments to make real-time calculations and deliver the information in an appropriate format.

Smartphone-based applications offer a solution for the uptake of VIS sampling plans in decisionmaking. We envision an app that will carry out the necessary calculations required for VIS sampling, as well as allow farmers to input their perceived risk and have a threshold calculated for them to base their decisions off of. We further propose that, integrated with machine learning, such an application could include user feedback to adjust these thresholds based on their own assessment of the decisions they made in the past. Even though most applications used in agriculture nowadays are designed for individual growers (Eichler Inwood and Dale 2019), such an app could also be used collectively by growers in such a way at information about produce price, pest incidence in an area or other risk conditions is used to better learn, and calculate thresholds in real time based off collective and historical data supplied by farmer's own experiences. By analyzing all the information gathered from various growers mathematically by the app, rather than subjectively by growers or external sources, alarm behavior could be avoided and could increase the uptake of IPM at regional scales. Although the use of applications in agriculture is incipient, a recent study by Bonke et al (2018) shows that in Germany, 93\% of farmers who participated in the study use agricultural apps, and $82 \%$ are willing to pay for applications which will help them better manage their crops. These results show that farmers are willing to use technology to help them in their decision-making process. Even though there is no information on the uptake of apps in Latin American agriculture, it is safe to assume that it will increase in the coming years, particularly if farmers are involved in their development firsthand.

\section{Conclusions}

Sustainability requires farmers to shift from strongly preventive agriculture, based on low pest tolerance and an overuse of inputs, to one that is corrective, based on objective information. The 
main difficulty in achieving this change is that farmers have an aversion to risk since their livelihood depends on the quality of their harvest. Even though oftentimes quality or quantity won't be affected by low pest populations, perceived risk outweighs rational decision-making. However, risk aversion may best be dealt with through experience. As such, sampling plans should not be based on a static threshold for decision-making which farmers had no hand in developing and may not apply to their current conditions, but rather, on dynamic thresholds based on the risk farmers' estimate themselves. We show both through computer simulations of field data, and through human sampling, that VIS sampling plans, and particularly those based on counts, not only give the most accurate estimation of pest densities but are also not more labor intensive than traditional SPRT sampling methods. Since we now have the capacity to carry out the calculations required by VIS sampling easily in the field, the accuracy obtained from it will allow farmers to determine how close, or far, they are from a threshold which may vary according to their perceived risk. We propose that allowing farmers to experiment with using control measures in a more informed way will change their own perception of risk and reduce pesticide applications. If farmers experiment in low-risk situations (moments with low market price or low pest populations) and begin to see for themselves that they may reduce pesticide applications, they will become more prone to taking risks under higher-risk situations. Once farmers realize that zero tolerance policies, such as that established for TLM in greenhouse tomato crops, are actually harming their income through the unnecessary use of agricultural inputs will we be able to move towards a future of sustainable food production.

\section{Acknowledgements}

We are grateful to Diego Fernando Sanchez, Marcela Preciado and Victor Pulido (AGROSAVIA) who provided valuable support collecting the data, and to Patricia Corredor, Edison Corredor, Atalivar Ramirez, Ernesto Cubides, Jesus Heredia, y Maria H. Pardo who allowed us to work in their greenhouses to carry out part of this study. This study was funded by the Ministerio de Agricultura y Desarrollo Rural de Colombia with government funds allocated to the Corporación Colombiana de Investigación Agropecuaria - AGROSAVIA (Grant No. 1000347 to DFR). Research support was provided by government funds assigned to AGROSAVIA. The authors assume full responsibility for the interpretation of results and ideas presented in this manuscript.

\section{Conflict of Interests}

The authors declare that there are no conflicts of interest 


\section{Author contribution}

413 Conceptualization, DFR; methodology, DFR, FB-E, HFR-T; software, DFR; formal analysis, DFR;

414 investigation, DFR, FB-E, HFR-T, LM-R; validation, HFR-T, LM-R; writing - original draft

415 preparation, DFR, FB-E; writing - review and editing, DFR, FB-E, HFR-T, LM-R; visualization,

416 DFR, FB-E, HFT-T; supervision, DFR.

\section{Data Availability}

418 The datasets generated during and/or analyzed during the current study are available from the 419 corresponding author on reasonable request.

420

421

422

423

424

425

426

427

428

429

430

431

432

433

434

435

436

437

438

439

440

441

442

443

\section{References}

Barberis NC (2013) Thirty Years of Prospect Theory in Economics: A Review and Assessment. Journal of Economic Perspectives 27 (1):173-196. doi:10.1257/jep.27.1.173

Barzman M, Bàrberi P, Birch ANE, Boonekamp P, Dachbrodt-Saaydeh S, Graf B, Hommel B, Jensen JE, Kiss J, Kudsk P (2015) Eight principles of integrated pest management. Agronomy for Sustainable Development 35 (4):1199-1215. doi:10.1007/s13593-015-03279

Binns MR, Nyrop JP, Werf Wvd (2000) Sampling and monitoring in crop protection : the theoretical basis for developing practical decision guides. CABI Pub., Wallingford, Oxon, UK; New York, N.Y.

Bonke V, Fecke W, Michels M, Musshoff O (2018) Willingness to pay for smartphone apps facilitating sustainable crop protection. Agronomy for Sustainable Development 38 (5):51. doi:10.1007/s13593-018-0532-4

Cassino P, Perusso J, Rego L, HN S (1995) Proposta metodológica de monitoramento de pragas emtomateiro estaqueado. Anais da Sociedade Entomológica do Brasil 24:279-285

Cocco A, Serra G, Lentini A, Deliperi S, Delrio G (2015) Spatial distribution and sequential sampling plans for Tuta absoluta (Lepidoptera: Gelechiidae) in greenhouse tomato crops. Pest Management Science 71 (9):1311-1323. doi:10.1002/ps.3931

Donado MdP, Soto M, Camelo M, Rincon DF, Espitia E (2017) Riesgos determinados sobre los principales contaminantes químicos y microbiológicos en tomate cultivado bajo condiciones protegidas en los departamentos de Cundinamarca, Boyacá y Antioquia. Informe final de meta: Levantamiento de información primaria para construcción de línea base. Corporación Colombiana de investigación agropecuaria (AGROSAVIA), Mosquera (Cundinamarca) 
444

445

446

447

448

449

450

451

452

453

454

455

456

457

458

459

460

461

462

463

464

465

466

467

468

469

470

471

472

473

474

475

476

Eichler Inwood SE, Dale VH (2019) State of apps targeting management for sustainability of agricultural landscapes. A review. Agronomy for Sustainable Development 39 (1):8. doi:10.1007/s13593-018-0549-8

Feola G, Binder CR (2010) Identifying and investigating pesticide application types to promote a more sustainable pesticide use. The case of smallholders in Boyacá, Colombia. Crop Protect 29 (6):612-622. doi:10.1016/j.cropro.2010.01.008

Fraser EDG, Campbell M (2019) Agriculture 5.0: Reconciling Production with Planetary Health. One Earth 1 (3):278-280. doi:10.1016/j.oneear.2019.10.022

Haddi K, Berger M, Bielza P, Cifuentes D, Field LM, Gorman K, Rapisarda C, Williamson MS, Bass C (2012) Identification of mutations associated with pyrethroid resistance in the voltage-gated sodium channel of the tomato leaf miner (Tuta absoluta). Insect Biochemistry and Molecular Biology 42 (7):506-513. doi:https://doi.org/10.1016/j.ibmb.2012.03.008

Hoy CW (1991) Variable-Intensity Sampling for Proportion of Plants Infested with Pests. Journal of Economic Entomology 84 (1):148-157. doi:10.1093/jee/84.1.148

Hoy CW, Jennison C, Shelton AM, Andaloro JT (1983) Variable-intensity sampling - a new technique for decision-making in cabbage pest-management. Journal of Economic Entomology 76 (1):139-143

Keller G, Novák V, Willems T (2019) A note on optimal experimentation under risk aversion. Journal of Economic Theory 179:476-487. doi:https://doi.org/10.1016/j.jet.2018.11.006

Lybbert TJ, Magnan N, Gubler WD (2016) Multidimensional Responses to Disease Information: How Do Winegrape Growers React to Powdery Mildew Forecasts and To What Environmental Effect? American Journal of Agricultural Economics 98 (2):383-405. doi:10.1093/ajae/aav097

Morse S (2008) IPM: Ideals and realities in developing countries. In: Radcliffe EB, Cancelado RE, Hutchison WD (eds) Integrated Pest Management: Concepts, Tactics, Strategies and Case Studies. Cambridge University Press, Cambridge, pp 458-470. doi:10.1017/CBO9780511626463.016

Naranjo SE, Chu CC, Henneberry TJ (1996) Economic injury levels for Bemisia tabaci (Homoptera: Aleyrodidae) in cotton: Impact of crop price, control costs, and efficacy of control. Crop Protection 15 (8):779-788

Parsa S, Morse S, Bonifacio A, Chancellor TCB, Condori B, Crespo-Pérez V, Hobbs SLA, Kroschel J, Ba MN, Rebaudo F, Sherwood SG, Vanek SJ, Faye E, Herrera MA, Dangles O (2014) Obstacles to integrated pest management adoption in developing countries. 
Proceedings of the National Academy of Sciences 111 (10):3889-3894. doi:10.1073/pnas.1312693111

Peterson RKD, Higley LG, Pedigo LP (2018) Whatever Happened to IPM? American Entomologist 64 (3):146-150. doi:10.1093/ae/tmy049

Plant RE (1986) Uncertainty and the Economic Threshold. Journal of Economic Entomology 79 (1):1-6. doi:10.1093/jee/79.1.1

Pratt JW, Raiffa H, Schlaifer R (1995) Introduction to Statistical Decision Theory. McGraw Hill, R Core Team (2018) R: A Language and Environment for Statistical Computing. Version 3.5.1. "Feather Spray" edn. R Foundation for Statistical Computing, Vienna, Austria

Rincon DF, Vasquez DF, Rivera-Trujillo HF, Beltrán C, Borrero-Echeverry F (2019) Economic injury levels for the potato yellow vein disease and its vector, Trialeurodes vaporariorum (Hemiptera: Aleyrodidae), affecting potato crops in the Andes. Crop Protection 119:52-58. doi:https://doi.org/10.1016/j.cropro.2019.01.002

Rykiel EJ (1996) Testing ecological models: The meaning of validation. Ecological Modelling 90 (3):229-244. doi:10.1016/0304-3800(95)00152-2

Silva GA, Picanço MC, Bacci L, Crespo ALB, Rosado JF, Guedes RNC (2011) Control failure likelihood and spatial dependence of insecticide resistance in the tomato pinworm, Tuta absoluta. Pest Management Science 67 (8):913-920. doi:10.1002/ps.2131

Stonehouse JM (1995) Pesticides, Thresholds and the Smallscale Tropical Farmer. International Journal of Tropical Insect Science 16 (3):259-262. doi:10.1017/s1742758400017264

Torres JB, Faria CA, Evangelista WS, Pratissoli D (2001) Within-plant distribution of the leaf miner Tuta absoluta (Meyrick) immatures in processing tomatoes, with notes on plant phenology. International Journal of Pest Management 47 (3):173-178. doi:10.1080/02670870010011091

Tversky A, Kahneman D (1981) The framing of decisions and the psychology of choice. Science 211 (4481):453-458. doi:10.1126/science.7455683

Viscusi WK (1997) Alarmist Decisions with Divergent Risk Information. The Economic Journal 107 (445):1657-1670

Wald A (1945) Sequential Tests of Statistical Hypotheses. The Annals of Mathematical Statistics 16 (2):117-186. doi:10.2307/2235829 


\section{Figures}

A.

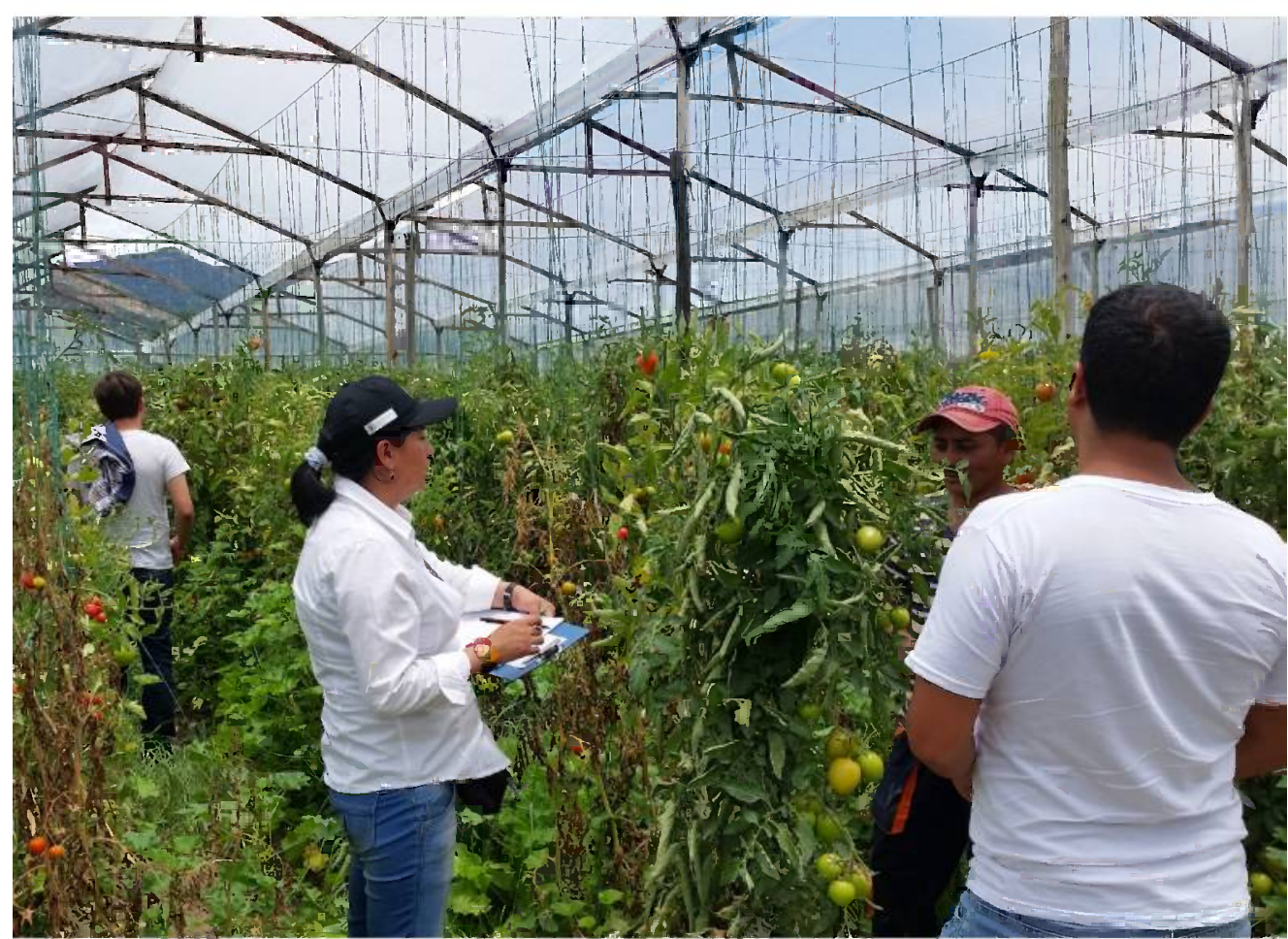

B.

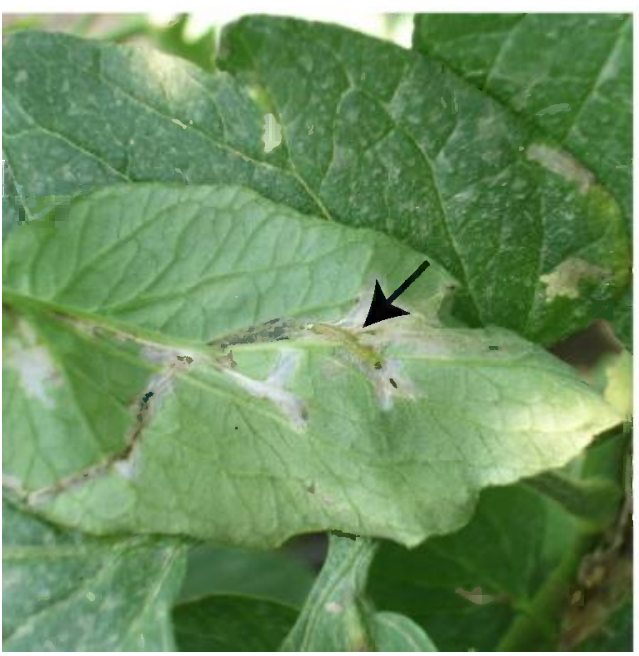

c.

Figure 1. Participatory sampling of Tuta absoluta larvae in greenhouses of Boyacá, Colombia.

510 Growers were instructed to carry out the sampling plans (A) by inspecting leaves (B) and fruits (C)

511 for damage or insects. Arrows show TLM larvae on leaves and fruits. Pictures taken by the authors. 
A.

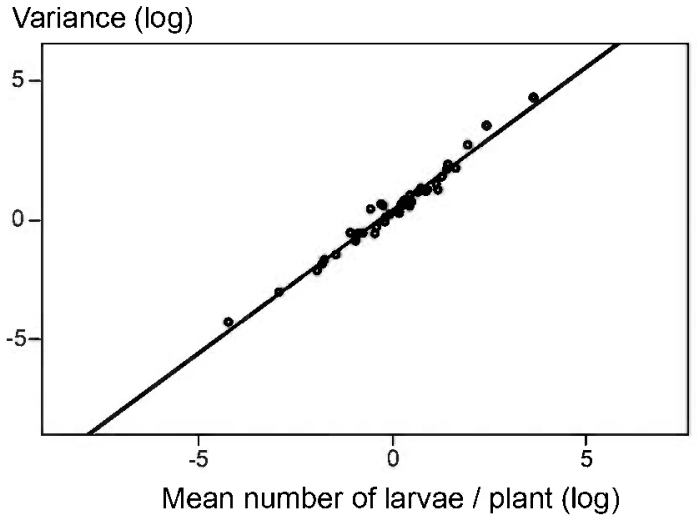

B. Proportion of infested plants

B. $(>0$ larvae)

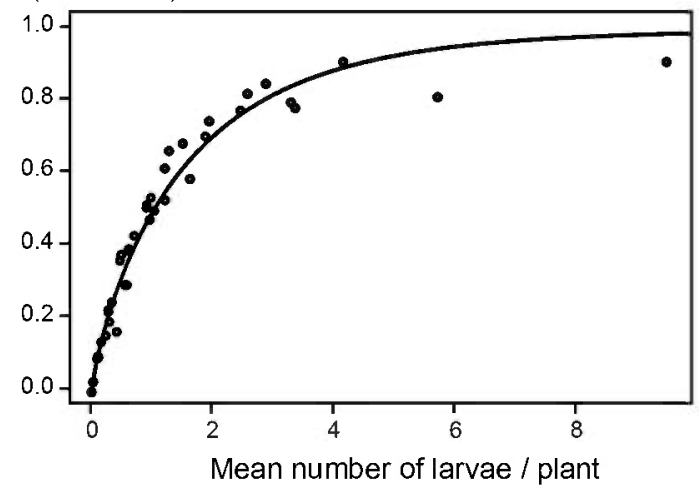

515 Figure 2. Aggregation pattern of Tuta absoluta larvae found in greenhouse tomato crops $(\mathrm{N}=40)$.

516 A) Variance-mean relationship. B) Proportion of infested plants as a function of mean number of 517 larvae per plant. 
A.

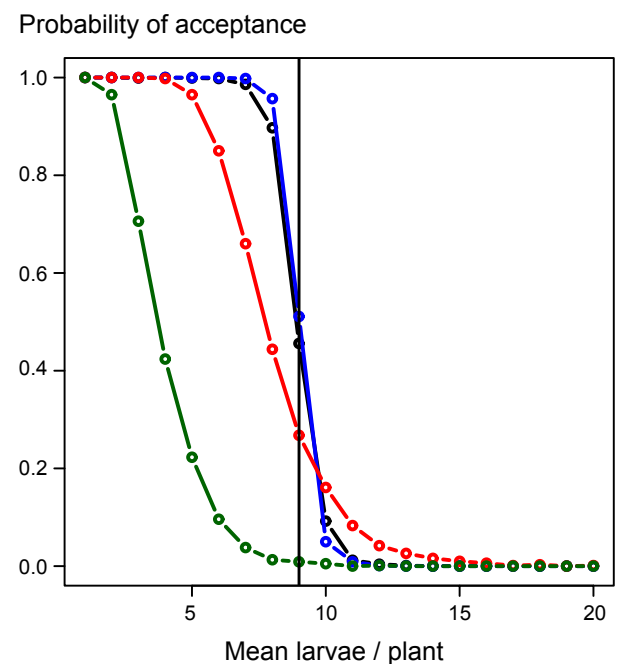

C.

Average number of samples

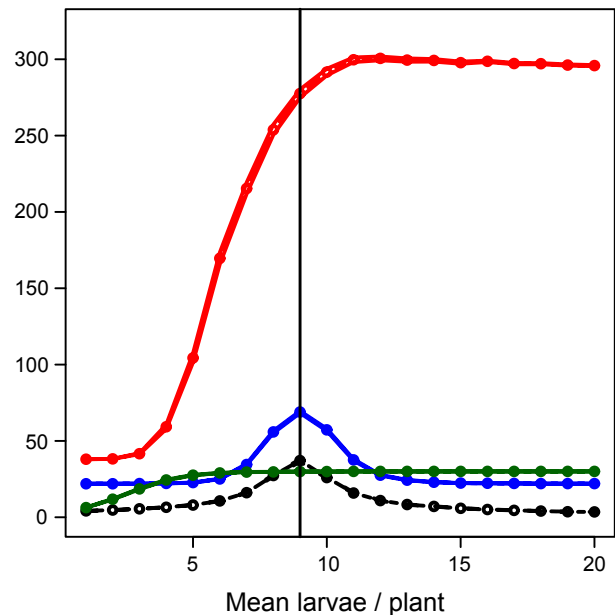

E.

Root mean squared error

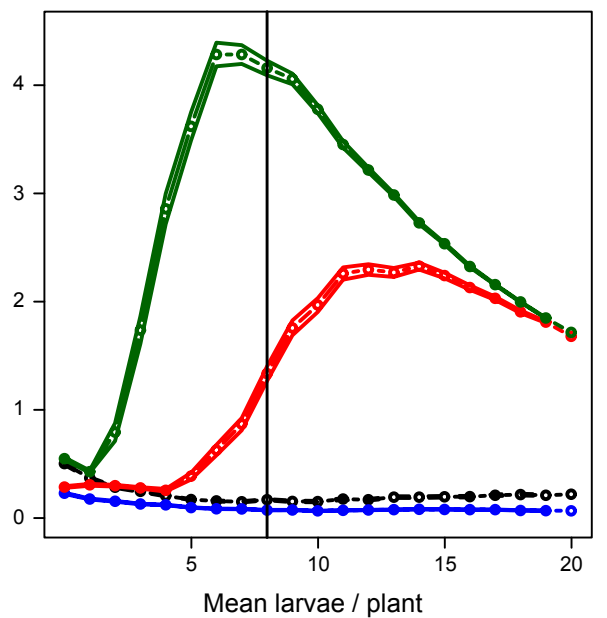

B.

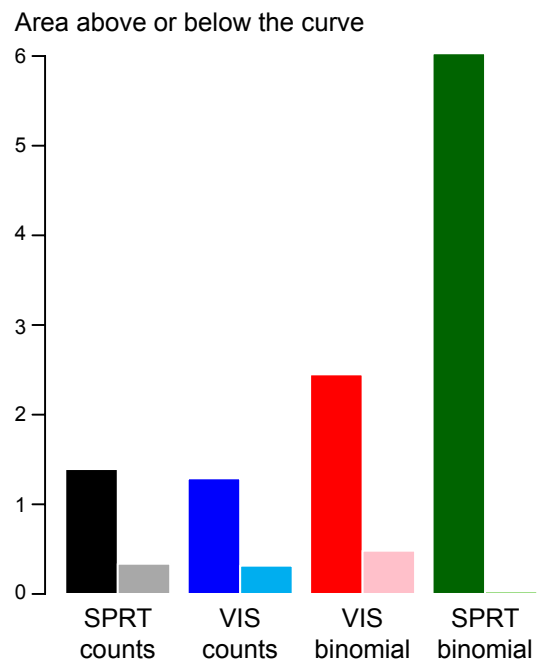

D.

Area under the curve

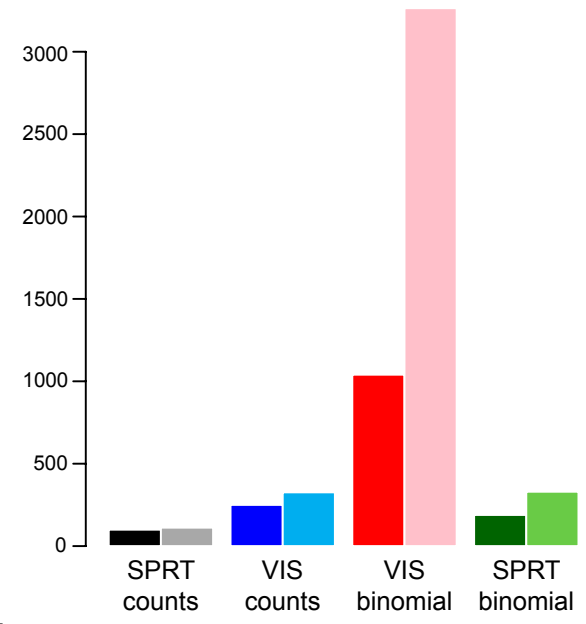

F.

Area under the curve

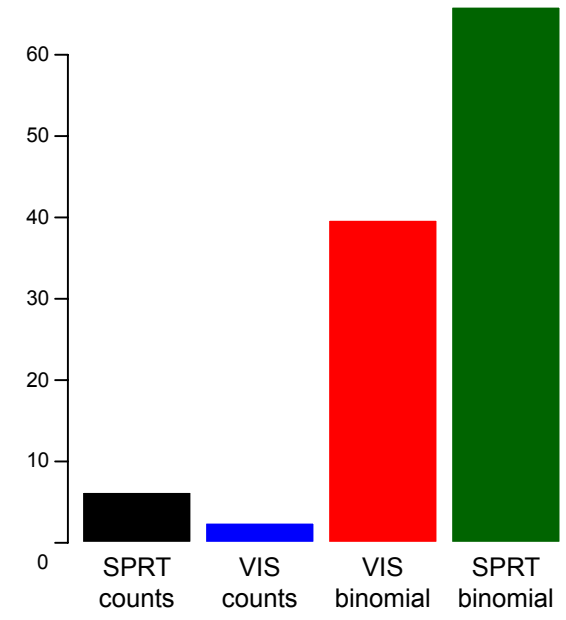


519 Figure 3. Evaluation of sampling plans (sequential probability ratio test [SPRT] by counts [black]

520 and binomial [red], and variable-intesity sampling [VIS] by counts [blue] and binomial [green])

521 using computer simulations. A first comparison shows differences in operating characteristic

522 curves (A) and the area above (dark colors) and under (light colors) the curves, before and after the

523 decision threshold, respectively (B). There were also diffrences in the average number of samples

524 required as a function of mean larvae per plant (C) and in the magnitude of the area under the

525 curves, before (dark colors) and after (light colors) the decision threshold, respectively (D). The last

526 comparison shows the differences in accuracy to estimate the population density of Tuta absoluta,

527 using the root mean squared error as a function of mean larvae per plant (E), and the area under the

528 curves (D).

A.

Mean larvae / plant

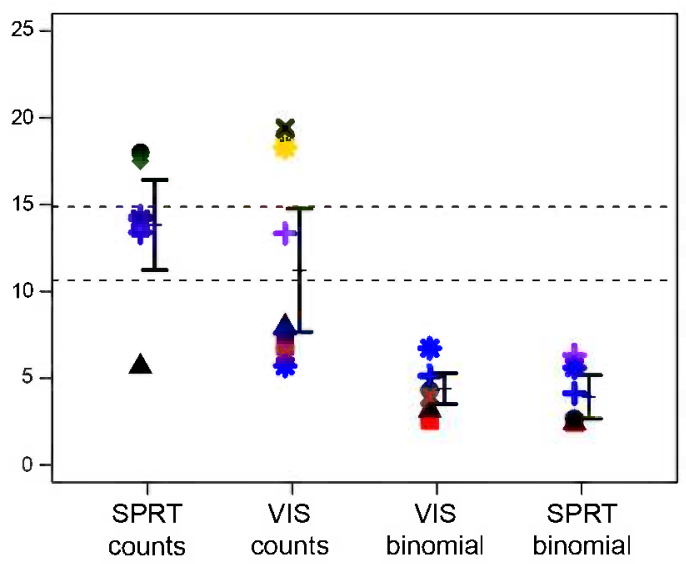

C. Number of samples

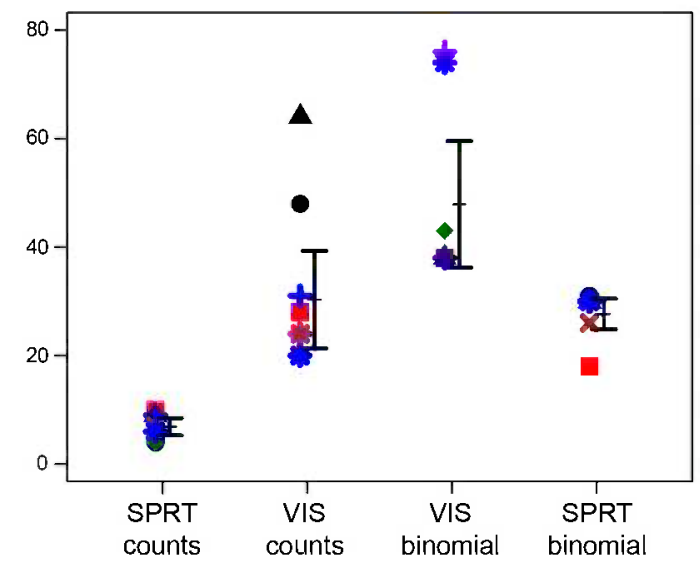

B.

Root mean squared error
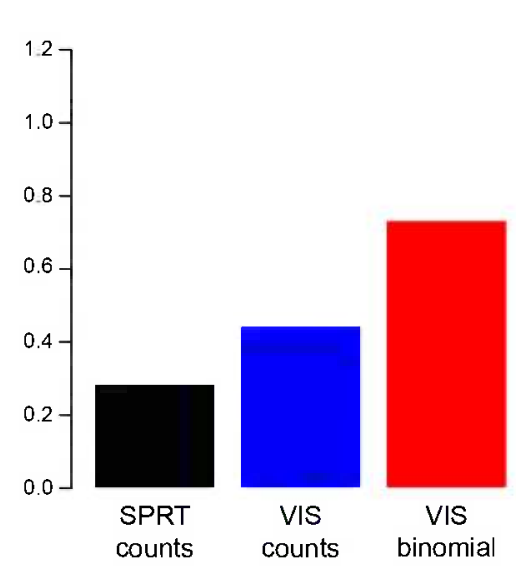

VIS

binomial

SPRT binomial

D.

Time (min)

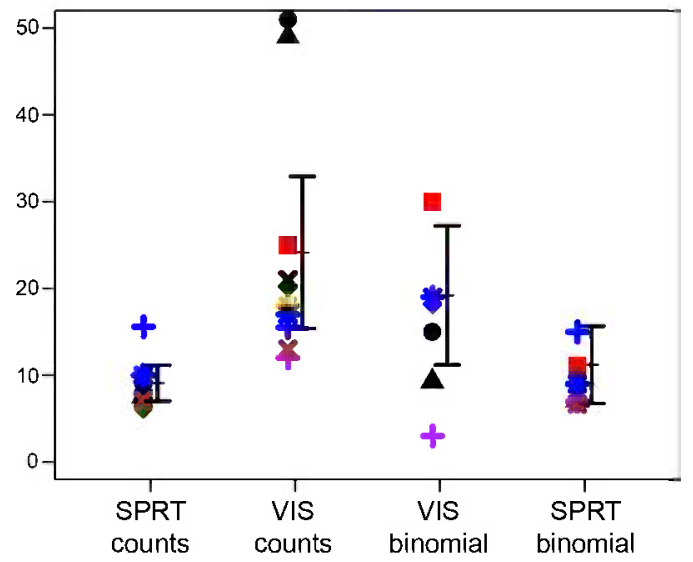

Figure 4. Evaluation of sampling plans in a field trial with human subjects. Each point type in A, C and D represents a different human individual. Plot A shows the comparison in precision and

532 accuracy of sampling plans estimating Tuta absoluta population density, and B the root mean 
533 squred error of each. Plots $\mathrm{C}$ and D show show the comparison in number of samples required and 534 time (in minutes), respectively. 